\title{
Correction to: Stochastic Higher Spin Vertex Models on the Line
}

\author{
Ivan Corwin ${ }^{1,2,3}$, Leonid Petrov ${ }^{4,5}$ (]) \\ 1 Department of Mathematics, Columbia University, 2990 Broadway, New York, NY 10027, USA. \\ E-mail: ivan.corwin@gmail.com \\ 2 Clay Mathematics Institute, 10 Memorial Blvd. Suite 902, Providence, RI 02903, USA \\ 3 Institut Henri Poincaré, 11 Rue Pierre et Marie Curie, 75005 Paris, France \\ 4 Department of Mathematics, University of Virginia, 141 Cabell Drive, Kerchof Hall, \\ P.O. Box 400137, Charlottesville, VA 22904-4137, USA. E-mail: lenia.petrov@ gmail.com \\ 5 Institute for Information Transmission Problems, Bolshoy Karetny per. 19, Moscow 127994, Russia
}

Received: 7 June 2019 / Accepted: 18 June 2019

Published online: 6 August 2019 - (C) Springer-Verlag GmbH Germany, part of Springer Nature 2019

\section{Correction to: Commun. Math. Phys. 343, 651-700 (2016) https://doi.org/10.1007/s00220-015-2479-5}

This is an erratum to the paper [CP16]. The aim of this note is to address two separate errors in the paper. We are very grateful to Yier Lin for pointing these out to us.

\section{Finite Vertical Spin Plancherel Identities}

The first error propagates from the earlier work [BCPS15]. It deals with the Plancherel identity for the Bethe ansatz eigenfunctions in the case of finite vertical spin (in our notation, $v=q^{-I}$, where $I \in \mathbb{Z}_{\geq 1}$ ). This identity is formulated in Proposition A.3 of [CP16]. The case $I=1$ of this identity is the Plancherel identity for the ASEP / six vertex model eigenfunctions, and it was established by Tracy and Widom in [TW08]. For $I \geq 2$, it is not clear whether this Plancherel identity is correct, and how to establish it. The claim in Proposition A.3 of [CP16], therefore, is not justified and should be removed from the paper.

The paper [CP16] also references the earlier work [BCPS15] where the identity for $I=1$ was claimed to follow from the general higher spin (i.e., general $v$ ) Plancherel identity. This claim is also false, as explained in detail in erratum [BCPS18].

\section{Duality (Theorem 2.23)}

All four dualities of Theorem 2.23 in [CP16] (Theorem 2.22 in the arXiv v1 version) are false. Here, we reproduce a counterexample provided to us by Yier Lin to the fourth of these dualities. 
Recall from [CP16, Definition 2.5], the state spaces for $k \in \mathbb{Z}_{\geq 1}$ :

$$
\begin{aligned}
\mathbb{G}^{k} & :=\left\{\vec{g}=\left(\ldots, g_{-1}, g_{0}, g_{1}, \ldots\right): g_{i} \in \mathbb{Z}_{\geq 0} \text { for all } i, \text { and } \sum g_{i}=k\right\} ; \\
\mathbb{W} \mathbb{Y}^{k} & :=\left\{\vec{n}=\left(n_{1} \geq \ldots \geq n_{k}\right): n_{i} \in \mathbb{Z} \text { for all } i\right\} .
\end{aligned}
$$

These spaces are in bijection with each other. Namely, we view elements of both $\mathbb{G}^{k}$ and $\mathbb{W} \mathbb{Y}^{k}$ as $k$-particle configurations on $\mathbb{Z}$, where $g_{i}$ is the number of particles at site $i$, and $n_{j}$ is the location of the $j$-th particle from the right.

The higher spin zero range process $\vec{g}(t)$, with discrete time $t$, is defined in [CP16, Definition 2.6]. It acts in the coordinates $\mathbb{G}^{k}$ by randomly moving particles to the right, in a left-to-right update manner, according to certain local stochastic vertex weights $L_{\alpha}^{(1)}$ depending on three parameters $q, \alpha, v$.

Let $\vec{n}(t)$ be the same higher spin zero range process, but with particles moving to the left, and written in the $\mathbb{W}^{k}{ }^{k}$ coordinates.

Theorem 2.23 in [CP16] claims that $\vec{g}(t)$ and $\vec{n}(t)$ are dual with respect to the functional

$$
\widehat{\mathrm{G}}_{\mathrm{n}}(\vec{g}, \vec{n})=\prod_{i=1}^{k}\left[g_{n_{i}}\right]_{q} q^{-\mathrm{N}_{n_{i}}^{\downarrow}(\vec{g})},
$$

where $\mathrm{N}_{n}^{\downarrow}(\vec{g})=\sum_{x \leq n} g_{x}$, and $[m]_{q}:=\frac{1-q^{m}}{1-q}$ (note that $[0]_{q}=0$ ). The (one time step) duality means that for all initial states $\vec{g}(0) \in \mathbb{G}^{l}$ and $\vec{n}(0) \in \mathbb{W} \mathbb{Y}^{k}$, one has

$$
\mathbb{E}^{\vec{g}(0)}\left[\widehat{\mathrm{G}}_{\mathrm{n}}(\vec{g}(1), \vec{n}(0))\right]=\mathbb{E}^{\vec{n}(0)}\left[\widehat{\mathrm{G}}_{\mathrm{n}}(\vec{g}(0), \vec{n}(1))\right]
$$

The counterexample will show that (1) is false.

Let $l=1$ and $k=2$, and the initial states be:

- a single particle at 0 for $\vec{g}(0)$;

- one particle at 0 and one particle at 1 for $\vec{n}(0)$.

The left-hand side of (1) is zero because it is a product of two factors containing $\left[g_{0}(1)\right]_{q}$ and $\left[g_{1}(1)\right]_{q}$, and at least one of these quantities is zero.

In the right-hand side of (1), the quantity under the expectation is nonzero iff $\vec{n}(1)=$ $(0,0)$. In this case, we have $\widehat{G}_{n}(\vec{g}(0), \vec{n}(1))=q^{-2}$, and the probability of the transition $(0,1) \rightarrow(0,0)$ in $\vec{n}(t)$ can be found from the vertex weights [CP16, (2.1)] to be

$$
\frac{\alpha(1-q)(1-q \nu)}{(1+\alpha)^{2}} .
$$

Therefore, the right-hand side of (1) is $\frac{\alpha(1-q)(1-q v)}{(1+\alpha)^{2}} q^{-2}$. This quantity cannot be zero unless $\alpha=0, q=1$, or $q v=1$. The first two cases trivialize the stochastic processes. The third case corresponds to the degeneration of the process to the stochastic six vertex model. This type of duality for the stochastic six vertex model was established recently in [Lin19]. 


\section{Updated Version}

We have prepared an updated version [CP19] of the paper [CP16] which removes these incorrect statements:

- The discussion of finite vertical spin Plancherel identities from Appendix A;

- The parts of Sections 5.3-5.5 using the finite vertical spin Plancherel identities;

- Theorem 2.23 and parts of Corollary 3.3 using it;

- Parts of Sections 5.3-5.5 involving the incorrect duality from Theorem 2.23.

There is a note in place of former Theorem 2.23 referencing this erratum. The other main statements of the paper (the definition of new stochastic particle systems, duality relations for them, and contour integral observables) are not affected. The updated version also contains journal references for the cited works, and minor corrections throughout.

Publisher's Note Springer Nature remains neutral with regard to jurisdictional claims in published maps and institutional affiliations.

\section{References}

[BCPS15] Borodin, A., Corwin, I., Petrov, L., Sasamoto, T.: Spectral theory for interacting particle systems solvable by coordinate Bethe ansatz. Commun. Math. Phys. 339(3), 1167-1245 (2015)

[BCPS18] Borodin, A., Corwin, I., Petrov, L., Sasamoto, T.: Erratum to "Spectral theory for interacting particle systems solvable by coordinate Bethe ansatz" (2018) Submitted. https://storage.lpetrov. cc/research_files/Petrov-publ/erratum_1407.pdf

[CP16] Corwin, I., Petrov, L.: Stochastic higher spin vertex models on the line. Commun. Math. Phys. 343(2), 651-700 (2016). arXiv:1502.07374 [math.PR]

[CP19] Corwin, I., Petrov, L.: Stochastic higher spin vertex models on the line. Updated version (2019) arXiv:1502.07374v2 [math.PR]. https://arxiv.org/abs/1502.07374v2

[Lin19] Lin, Y.: Markov duality for stochastic six vertex model (2019) arXiv preprint arXiv:1901.00764 [math.PR]

[TW08] Tracy, C., Widom, H.: Integral formulas for the asymmetric simple exclusion process. Commun. Math. Phys 279, 815-844 (2008). arXiv:0704.2633 [math.PR]. Erratum: Commun. Math. Phys. 304, 875-878 (2011)

Communicated by A. Borodin 